\title{
In Silico Prediction of the Chemical Block of Human Ether-a-Go-Go-Related Gene (hERG) K+ Current
}

\author{
Atsushi INANOBE ${ }^{1,2}$, Narutoshi KAMIYA ${ }^{2,3}$, Shingo MURAKAMI ${ }^{1,2}$, Yoshifumi FUKUNISHI ${ }^{4}$, \\ Haruki NAKAMURA ${ }^{2,3}$, and Yoshihisa KURACHI ${ }^{1,2}$ \\ ${ }^{1}$ Division of Molecular and Cellular Pharmacology, Department of Pharmacology, Graduate School of Medicine, ${ }^{2}$ the Center \\ for Advanced Medical Engineering and Informatics, ${ }^{3}$ Laboratory of Protein Informatics, Institute for Protein Research, Osaka \\ University, Osaka; and ${ }^{4}$ Biomedicinal Information Research Center, National Institute of Advanced Industrial Science and \\ Technology, Tokyo, Japan
}

\begin{abstract}
A variety of compounds with different chemical properties directly interact with the cardiac repolarizing $\mathrm{K}^{+}$channel encoded by the human ether-a-go-go-related gene (hERG). This causes acquired forms of QT prolongation, which can result in lethal cardiac arrhythmias, including torsades de pointes one of the most serious adverse effects of various therapeutic agents. Prediction of this phenomenon will improve the safety of pharmacological therapy and also facilitate the process of drug development. Here we propose a strategy for the development of an in silico system to predict the potency of chemical compounds to block hERG. The system consists of two sequential processes. The first process is a ligand-based prediction to estimate half-maximal concentrations for the block of compounds inhibiting hERG current using the relationship between
\end{abstract}

chemical features and activities of compounds. The second process is a protein-based prediction that comprises homology modeling of $h E R G$, docking simulation of chemical-channel interaction, analysis of the shape of the channel pore cavity, and Brownian dynamics simulation to estimate hERG currents in the presence and absence of chemical blockers. Since each process is a combination of various calculations, the criterion for assessment at each calculation and the strategy to integrate these steps are significant for the construction of the system to predict a chemical's block of hERG current and also to predict the risk of inducing cardiac arrhythmias from the chemical information. The principles and criteria of elemental computations along this strategy are described.

Key words: hERG, quantitative structure-activity relationship, molecular docking, Brownian dynamics simulation.

$\mathrm{Mary}_{\mathrm{a}}$ heart. The ventricular action potential possesses a long lasting plateau that is terminated by the activation of repolarizing $\mathrm{K}^{+}$currents, $I_{\mathrm{Kr}}$ and $I_{\mathrm{Ks}}$. The disturbance of these currents causes prolongation of the action potential duration and thus the QT interval of the electrocardiogram, and in many cases a worsening of transmural dispersion of repolarization [1-3]. These are the substrates for generation of life-threatening cardiac arrhythmias, including torsades de pointes. Many compounds inhibit $I_{\mathrm{Kr}}$, whose pore-forming subunit is the human ether-a-gogo-related gene (hERG) product. This can cause acquired forms of long QT syndrome [4-6]. The compounds exhibit a broad spectrum, including not only cardiac ion channel blockers, but also antipsychotic agents, antidepressant agents, antimicrobial agents, phosphodiesterase inhibitors, topoisomerase II inhibitors, and antagonists for $\mathrm{G}$ protein-coupled receptors, such as nonsedating antihistamine $\mathrm{H}_{1}$ receptor blockers and $\beta$ blockers [6]. The development of methods that could predict this phenomenon would improve the safety of pharmacological therapy and also facilitate the process of drug development.

Here we propose an in silico strategy to predict the potency of compounds to block hERG current. The strategy comprises two sequential processes: ligand- and proteinbased analyses (Fig. 1). The first, a ligand-based process, consists of steps necessary to obtain the half-maximal concentration for the block $\left(I C_{50}\right)$ of hERG. This assesses the effectiveness of compounds to inhibit hERG current. The putative hERG channel blockers are then characterized further with the second protein-based process. This process is an assembly of several steps to assess ion flux through hERG when the compound is bound in its pore. The first step is to dock compounds in the central cavity of the pore of hERG constructed by comparative model-

Received on Jul 31, 2008; accepted on Nov 22, 2008; released online on Nov 27, 2008; doi:10.2170/physiolsci.RV011408

Correspondence should be addressed to: Yoshihisa Kurachi, Division of Molecular and Cellular Pharmacology, Department of Pharmacology, Graduate School of Medicine, Osaka University, 2-2 Yamada-Oka, Suita, Osaka, 565-0871 Japan. Tel: +81-6-6879-3512, Fax: +81-6-6879-3519, E-mail: ykurachi@pharma2.med.osaka-u.ac.jp 
ing methods. The second consists of steps calculating the shape of the pore cavity for each docked model, averaging diameter of the pore of every model, and also selecting a model possessing a pore shape close to the average. The third process is then a Brownian dynamics (BD) simulation of ion permeation through the selected model. Except for the simulation, all steps have been reported previously and are publicly available. However, since they were generated for their own individual purposes, the strategy to integrate them serially and the criterion evaluation of each step to connect to the subsequent step are crucial for the construction of the system to predict the effect of compounds of interest on the hERG current. In this review, we describe the requirement and the principal of these processes and discuss the criterion for assessment at each step and the issue to be settled in the future.

\section{Ligand-based prediction system for the blockade of hERG current}

Statistical analyses of compounds possessing similar activity to modulate the function of a protein have the advantage of being able to predict molecular features common to the compounds without considering target protein structure [7, 8]. A broad spectrum of compounds different in size and shape can interact with hERG channels and inhibit ion flow [3]. This makes it difficult to recognize common properties of the compounds that block the channel. The ligand-based approach has been adopted to extract the features of hERG blockers in terms of inhibition of hERG current [9-13]. These methods include traditional two-dimensional (2D)-quantitative structure activity relationships (QSAR) [11, 14-16], 3D-QSAR [17-22], and classification [23-26] methods. These methods extract chemical properties such as steric, hydrophobic, and electronic properties from the compounds. The features are called descriptors [27, 28]. The methods compare the distribution of the descriptors from all of the compounds and then isolate the descriptors commonly observed among the compounds. Lastly, they generate a model for a mathematical relationship between biological activities of compounds and common descriptors as equation coefficients of QSAR. The activity of novel compounds can then be computed from these mathematical relationships.

In regard to $3 \mathrm{D}-\mathrm{QSAR}$ models, information about the spatial distribution is included in the characteristics of descriptors $[29,30]$. Before the extraction of descriptors, diverse conformations of molecules are generated. The calculation of 3D-QSAR models requires considerable computational power. But recent improvements in computation make the method straightforward. A conformational analyzer with molecular dynamics and sampling (CAMDAS) is one example of a program to derive compound conformations [31]. At present 3D-QSAR

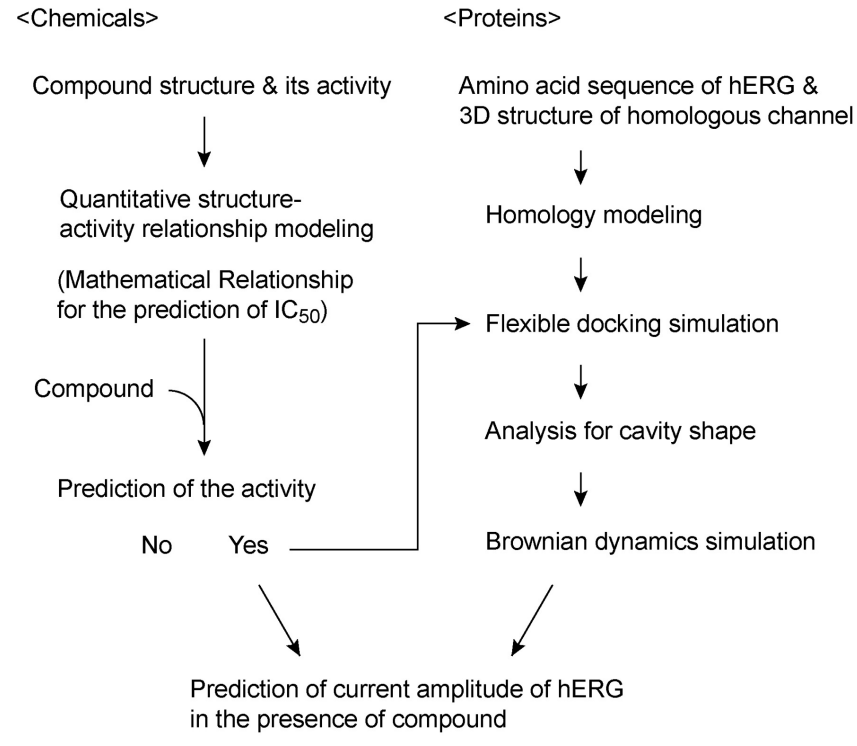

Fig. 1. Flowchart of the prediction system for the hERG current.

models could be generated by such programs as Catalyst (Accelrys, Inc., San Diego, USA), comparative molecular field analysis (CoMFA) [32], and comparative molecular similarity index analysis (CoMSIA) [33]. These programs generate a hypothetical pharmacophore as a set of descriptors distributed within a 3D space. Here we use the Catalyst program for the ligand-based estimation of $I C_{50}$ values of compounds.

\section{Strategy for the prediction of inhibitory activity of compounds on hERG}

2.1. Setting experimental conditions. To characterize the relationship between the compound and its activity, we surveyed the literature to list the biological activities of hERG blockers as $I C_{50}$ values (Fig. 2). There are two major expression systems for hERG current measurement: Xenopus oocytes and mammalian cells. But a block of hERG in Xenopus oocytes requires nearly a tenfold higher concentration of a compound than the hERG current expressed in mammalian cells [34, 35]. Diverse explanations could account for these differences. In this study we used $I C_{50}$ values of compounds measured in mammalian cell expression systems and isolated cardiac myocytes as indicated in the figure legend.

2.2. Generation of 3D-QSAR pharmacophore model. Chemical structures were generated using the editor sketcher in Discovery Studio 1.7 (Accelrys) and minimized the conformational energy to the closest local minimum using the CHARMm (Chemistry at Harvard molecular mechanics)-like force field included in the suite. Diverse conformations of each chemical were generated, and conformations whose energy was less than $20 \mathrm{kcal} / \mathrm{mol}$ compared to the most stable conformer were 
A<smiles>CC1=C(N=CCN(CCO)CCc2ccc([N+](=O)[O-])cc2)C(=O)N(C)C(=O)C1=O</smiles>

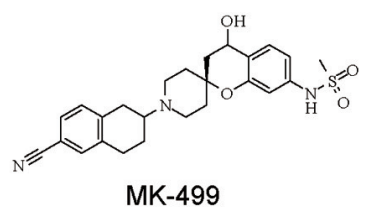<smiles>Cc1cccc(CCN2CCC(C(=O)c3ccc(C(C)(C)C)cc3)CC2)n1</smiles>

E-4031<smiles>COc1cc(N)cc(Cl)c1C(=O)NC1CCN(CCOc2ccc(F)cc2)CC1C</smiles>

cisapride<smiles></smiles>

dofetilide<smiles>CCCN=C(CCCCOC)c1ccc(C(F)(F)F)cc1</smiles>

fluvoxamine

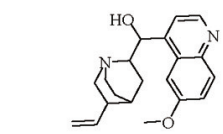

quinidine

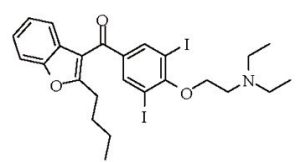

amiodarone
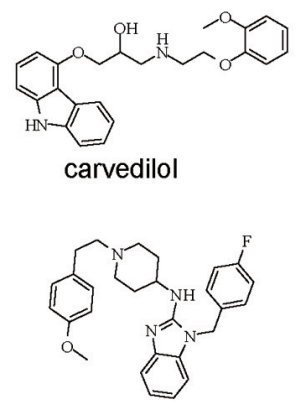

astemizole
B

\section{a}

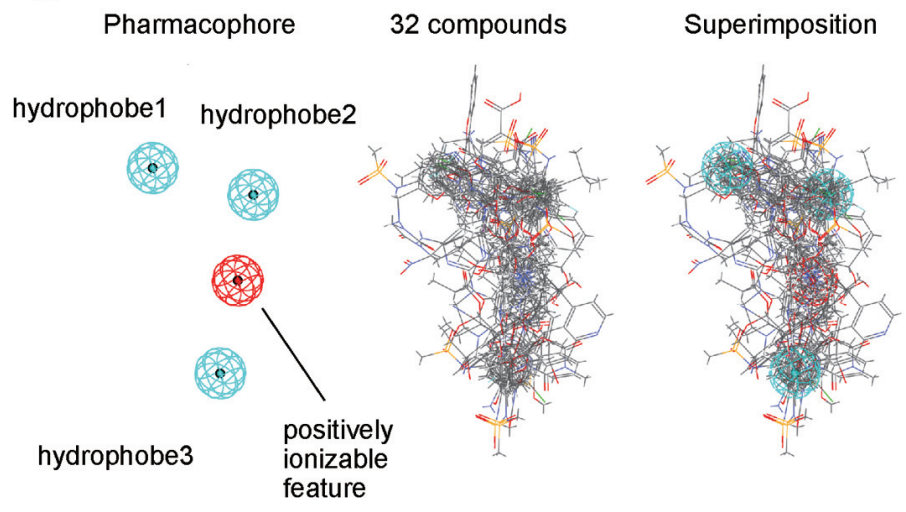

b

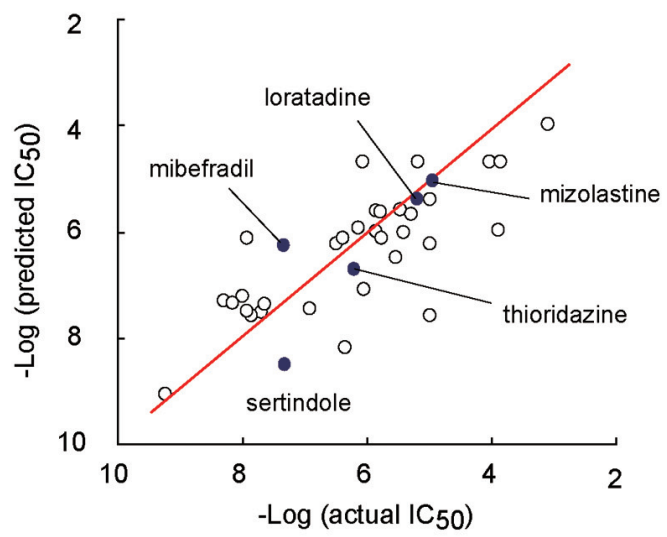

Fig. 2. Ligand-based prediction of the potency of compound to block $h E R G$ current. A. Chemical structure of hERG blockers. B. Generation of 3D-QSAR model. To generate 3D-QSAR, we used the following 32 compounds and $I C_{50}$ values for hERG expressed in mammalian cells: amiodarone $70 \mathrm{nM}$ [110], amitriptyline $10 \mu \mathrm{M}$ [111], astemizole $0.9 \mathrm{nM}$ [36], azimilide $0.61 \mu \mathrm{M}$ [112], bepridil $0.55 \mu \mathrm{M}$ [41], carvedilol $10 \mu \mathrm{M}$ [113], cisapride $14 \mathrm{nM}$ [114], clemastine $12 \mathrm{nM}$ [115], desipramine $1.4 \mu \mathrm{M}$ [20], disopyramide $7.2 \mu \mathrm{M}$ [116], dofetilide $90 \mathrm{nM}$ [117], dronedarone $59 \mathrm{nM}$ [110], E-4031 $7.7 \mathrm{nM}$ [118], fluoxetine $1.5 \mu \mathrm{M}$ [119], fluvoxamine $3.8 \mu \mathrm{M}$ [120], gatifloxacin $0.13 \mathrm{mM}$ [37], imipramine $3.4 \mu \mathrm{M}$ [121], ketoconazole $8.9 \mu \mathrm{M}$ [19], maprotiline $5.2 \mu \mathrm{M}$ [122], MK-499 $21 \mathrm{nM}$ [114], nifekalant $1.4 \mu \mathrm{M}$ [52], quinidine $0.41 \mu \mathrm{M}$ [123], prazosin $1.6 \mu \mathrm{M}$ [124], procainamide $0.14 \mathrm{mM}$ [125], propafenone $0.44 \mu \mathrm{M}$ [123], sotalol $150 \mu \mathrm{M}$ [126], terfenadine $9.0 \mathrm{nM}$ [114], trazodone $2.9 \mu \mathrm{M}$ [127], verapamil $0.44 \mu \mathrm{M}$ [41], and ziprasidone $0.12 \mu \mathrm{M}$ [128]. The $/ C_{50}$ values of almokalant $(50 \mathrm{nM})$ [108] and budipine $(0.85 \mu \mathrm{M})$ [129] measured with isolated ventricular myocytes from either rabbit or guinea pig were also included in the data set. (a) Common features of $h E R G$ blockers consist of three hydrophobes (hydrophobe 1-hydrophobe 3) and one positively ionizable feature. The hERG blockers could be aligned onto the pharmacophore assembly. (b) Measured $I C_{50}$ values of hERG blockers were plotted against estimated $I C_{50}$ values (open circles). The $I C_{50}$ values of compounds, mibefradil [41], sertindole [39], thioridazine [39], loratadine [40], and mizolastine [19], were predicted based on the 3D-QSAR (closed circles).

subjected to the following analysis. There are several methods available for generating 3D structures of molecules. All methods have limitations, since they generate the compound conformations in an in vacuo condition. We prepared $32 \mathrm{hERG}$ blockers and their $I C_{50}$ values (Fig. $2 \mathrm{~A}$ ). The $I C_{50}$ values ranged from $0.9 \mathrm{nM}$ (astemizole) [36] to $0.13 \mathrm{mM}$ (gatifloxacin) [37]. The 3D-QSAR models were generated by HipHop algorithm in the Catalyst program. The program first selects two of the most effective molecules among the test compounds, aligns their conformational models, and creates assemblies of descriptors common to the two molecules. After that, the program aligns the other compounds to each assembly and calculates an equation coefficient of each descriptor, weighing a distance from its ideal localization. Finally the program prints out assemblies having good correlations between measured and predicted $I C_{50}$ values as $3 \mathrm{D}-\mathrm{QSAR}$ pharmacophore hypotheses. Since hERG blockers possess diverse properties, we simply selected hydrophobic and positively ionizable features for the search of common chemical features of hERG blockers. Catalyst generated 10 hypotheses as pharmacophore models. These consist- 
ed of the assembly of three to five hydrophobic features combined with zero or one positively ionizable feature. The comparison between the input $I C_{50}$ values and the predicted $I C_{50}$ values indicated that the top-ranked hypothesis, which had three hydrophobic features and one positively ionizable feature, gave the best correlation between the two sets of values (Fig. 2Ba, left panel). The multiple coefficient of determination $\left(r^{2}\right)$ of 0.90 suggested that the generated model was statistically significant (Fig. 2Bb, open circles). The distances between all of the features were within $13.5 \AA$, and those between each hydrophobe and a positively ionizable feature ranged from $5.5 \AA$ to $8.9 \AA$. The ionizable feature locates $1.2 \AA$ apart from the plane of the three hydrophobes. Compound structures could be matched onto the feature assembly (Fig. 2Ba). The classical properties of hERG blockers, a basic nitrogen and an aromatic moiety, position at the positively ionizable feature and the three hydrophobes, respectively. This distribution of features of the hypothetical pharmacophore is similar to those reported by other investigators $[17,38]$.

Using this pharmacophore model, a validation test was performed by estimating $I C_{50}$ values of compounds known to block hERG, but that were not used to generate the model. Anti-psychotic drugs (thioridazine $\left(I C_{50} ; 0.191\right.$ $\mu \mathrm{M})[39]$ and sertindole $(0.003 \mu \mathrm{M})$ [39]), anti-histamine $\mathrm{H}_{1} \mathrm{R}$ antagonists (mizolastine $(8.9 \mu \mathrm{M})$ [19] and loratadine $(4.0 \mu \mathrm{M})[40])$, and the $\mathrm{Ca}^{2+}$ channel blocker (mibefradil $(1.43 \mu \mathrm{M})[41])$ were examined (Fig. 2Bb closed circles). The $I C_{50}$ values were calculated as $0.634,0.049,12,6.6$, and $0.047 \mu \mathrm{M}$, respectively, and the ratio of predicted $I C_{50}$ to measured $I C_{50}$ values were $3.3,16,1.3,1.6$, and 0.033 , respectively. These results imply that the $3 \mathrm{D}-\mathrm{QSAR}$ pharmacophore model possesses the properties of $\mathrm{hERG}$ blockers and is sufficient for the estimation of possible hERG blocking activity of a compound.

2.3. Issues and resolutions. A pharmacophore has been defined by the International Union of Pure and Applied Chemistry as "an ensemble of steric and electronic features that is necessary to ensure the optimal supramolecular interactions with a specific biological target and to trigger its biological response." Whereas computational analyses are able to point out the common features of molecules with the same pharmacological activity, these features may or may not be consistent with features that are known to be involved in the association between the compound and the target protein. For example, a side chain of Tyr652 on the S6 helix of hERG is reported to interact with the basic nitrogen center of the blocker based on cation- $\pi$ interaction [42]. Phe656 on the same helix is involved in the association with blockers through hydrophobic/ $\pi$-stacking interaction [42-44]. Furthermore, hydroxyl groups of Thr623 and Ser624 in a loop between the pore helix and the selectivity filter are thought to be involved in blocker-hERG association through hydrogen bonding [45]. These experimentally identified elements of interaction between compounds and hERG do not match with the features identified by in silico analysis, and the orientation of the pharmacophore within the central cavity of the hERG pore is also ambiguous.

Improvements of the ligand-based method can use a large data set to generate more precise volumes and regression coefficients for each pharmacophore feature. Or a combinatorial method with a classification $[23,24]$ based on a decision tree algorithm, which divides blockers into a hierarchy of homogeneous subgroups using the descriptors, can be used. By fitting the compounds to subgroup-specific pharmacophore models, the predicted activities of compounds have been reported to become closer to their actual activities $[9,16,46]$. An alternative would be to take into account the target protein. A structure-based screening method with homology models of the ion conduction unit of hERG to predict inhibitory activity of compounds is discussed below [47-52].

\section{Protein-based prediction system for hERG current}

More than 30,000 structures of soluble proteins have been reported, while about 150 unique structures of either whole or part of different membrane proteins have been deposited in the protein database [53]. But most of the membrane protein structures are derived from microbes (bacteria and archaea). This clearly limits structure-based computational approaches for the prediction of the association between a compound and a mammalian or human membrane protein. However, if an amino acid sequence shares more than $30 \%$ identity to one of the determined 3D structures in a database, the folding of the protein of interest can be constructed by comparative modeling methods with moderate accuracy [54]. The higher the degree of amino acid identity/similarity and the higher the resolution of the template structure both facilitate the accuracy of model building. Optimal alignment of amino acid sequences of the target protein and known structures is also crucial to generate an accurate target structure. The most widely used program to estimate 3D structure is MODELLER [55]. After the model is built, a refinement of the structure by force-field-based energy minimization is required to optimize the model. For the validation of the final structure, torsion angles, bond length, and van der Waals contacts can be assessed by the PROCHECK program [56].

The computational process to investigate how a molecule of interest interacts with its target receptor is called docking [8]. The 3D structures from a database or those generated by homology modeling can be used as receptors for docking analysis. The process is composed of two important steps: a search step for molecule binding spaces on the target protein and a scoring step to rank the binding positions [7]. Several docking methods have 
been developed, e.g., DOCK [57], AutoDock [58], FlexX [59], CDocker[60], GOLD [61], and Sievgene [62]. All methods basically repeat these two steps to explore molecule-protein complex combinations having better scores. The searching methods include docking algorithms by searching conformational space either before or after generating conformers of the molecule, or by searching favorable positions of fragments of the molecule in the binding site. The scoring is the estimation of the binding affinity represented by the sum of free energies of the elements of the interaction, such as hydrogen bonding, electrostatic interaction, van der Waals and dispersion interactions, and steric and solvation effects.

Crystal structures of proteins show us sophisticated molecular architecture at an atomic scale, but they are static and cannot provide structural information about how the protein alters its conformation during its function. Multiple conformational states of a protein in relation to its function can be modeled by a simulation technique known as molecular dynamics (MD) [63, 64]. But the application of this to an ion channel pore is not without its difficulties. The classical method for modeling ion permeation is the continuum theory of electrodiffusion expressed with Poisson-Nernst-Planck equations [65]. But since the mean field approximation requires a pore with a radius exceeding $16 \AA$ in the presence of the physiological concentration of salt, this method cannot be applied to simulate ion flow through typical ion channels $[66,67]$. In an MD simulation, the freedom of atoms is changed within a defined system in a stepwise manner. Newton's equations are applied to describe the mass and acceleration of all atoms, and an ensemble of these equations for atom motion yields a trajectory of each atom with the characteristics of its position, velocity, and acceleration. This method can be applied to the verification of ion selectivity $[68,69]$ and multi-ion configurations at the selectivity filter of $\mathrm{K}^{+}$channels $[68,70,71]$. However, there is an incompatibility between the time scales accessible to MD simulation and that of the permeation process. For example, for an ion channel with an open channel current amplitude of $5 \mathrm{pA}$, almost 30 ions permeate the pore every $1 \mu \mathrm{s}$. An MD simulation must repeat a million steps for $1 \mathrm{~ns}$ of simulation, and in general it can be run on a supercomputer for time scales of the order of $10 \mathrm{~ns}$. These scales are too short to follow ion conduction through an ion channel. A viable alternative to MD simulation is BD simulation, which enables simulations of the order of $10 \mu \mathrm{s}$ [72]. In BD simulation, water and protein are treated as continuous dielectric media with a rigid boundary between them [73]. Chaotic and diffusive properties of ion movement permit this simulation to calculate the trajectories of ions by integrating stochastic equations of motion (Langevin equation). The electric field and potential, which are effects of the dielectric boundary created by the water-protein interaction, as well as the reaction field, which represents the interaction of ions with charges on the channel wall, are calculated prior to the simulation and interpolate the required values during simulation [74-76]. Membrane potential, which is a driving force for $\mathrm{K}^{+}$ions passing through the pore, can be expressed with either a uniform electric field across the system or a grand canonical Monte Carlo method [77, 78]. Under these conditions, ion conduction has been examined for the bacterial KcsA channel [68, 70, 76, 79]. However, the treatment of water as a continuum medium, the simplification of the effect of the dielectric boundary on the ions, the neglect of local motions of amino acid side chains, and the application of constant field approximations are potential problems in BD simulation. Therefore both MD and BD simulations have their strong points and their weak points, and these approaches have been used to simulate different phases of ion channel function. Next we introduce the structure-based estimation of ion conduction of hERG in complex with compounds with sequential computational approaches.

\section{Strategy for the prediction of ion conductance of hERG in complex with compounds}

4.1. Generation of an open conformation model of hERG ion conduction unit. The hERG blockers are thought to interact with the central pore cavity of hERG $[3,9,12$, 13]. To simulate the association of compound and hERG with a protein-based approach, a $3 \mathrm{D}$ structure of hERG is required. In the absence of a crystal structure of hERG, a 3D structure must be constructed by computational approaches.

MacKinnon and co-workers have characterized several $\mathrm{K}^{+}$channels at the atomic level. They solved the crystal structures of a bacterial channel KcsA [80-82], a bacterial calcium-activated channel MthK [83], a bacterial voltage-gated channel KvAP [84], and a mammalian voltage-gated channel Kv1.2 as a complex with its accessory protein $\beta 2$ subunit in detergent [85] and a phospholipid bilayer [86]. Structures of a bacterial homologue of the inward rectifier $\mathrm{K}^{+}$channel KirBac1.1 [87] and a chimera between Kir3.1 and KirBac3.1 [88] have also been solved. All of these $\mathrm{K}^{+}$channel structures provide us with information on the general relationship between the structure and the function of $\mathrm{K}^{+}$channels. The $\mathrm{K}^{+}$channel is a tetrameric assembly. The center of four subunits is an ion conduction pathway surrounded by each structural element composed of two membrane-spanning helices (S5 and S6 helices, or outer and inner helices), one short pore helix, and a selectivity filter. The ion conduction pathway consists of three elements: the selectivity filter, the waterfilled central cavity, and the internal face of the pore constrained by four S6 helices. The selectivity filter consists of particular amino acid residues that are conserved in most $\mathrm{K}^{+}$channels and are crucial for ion selectivity. This region makes a narrow constraint in the ion conduction 
A

a

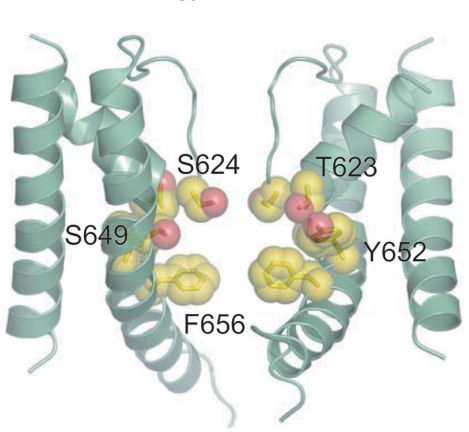

B

a Analysis for cavity shape compound-free hERG

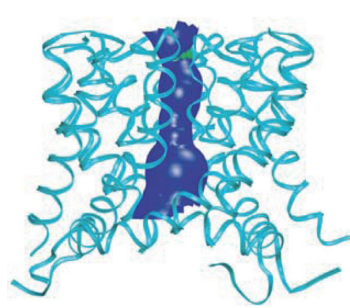
nifekalant-bound hERG

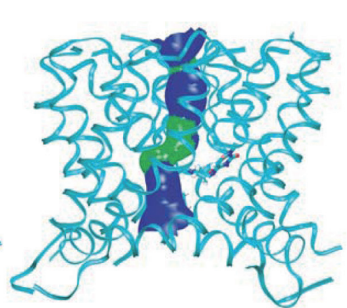

C

a Brownian dynamics simulation

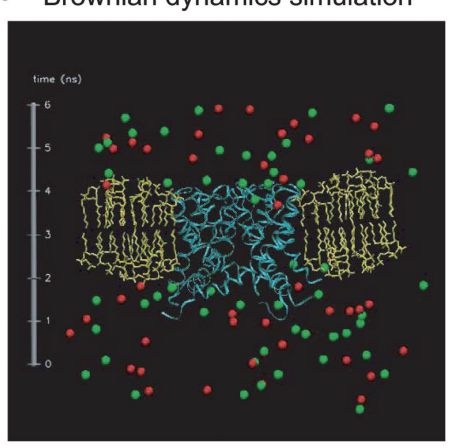

b

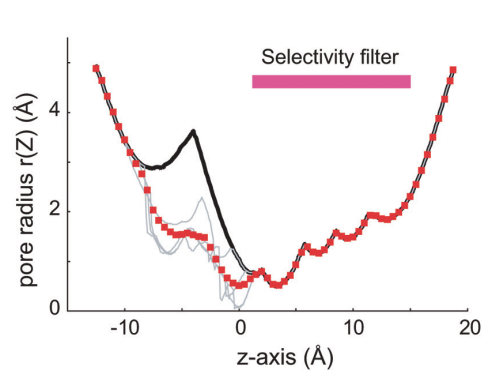

b

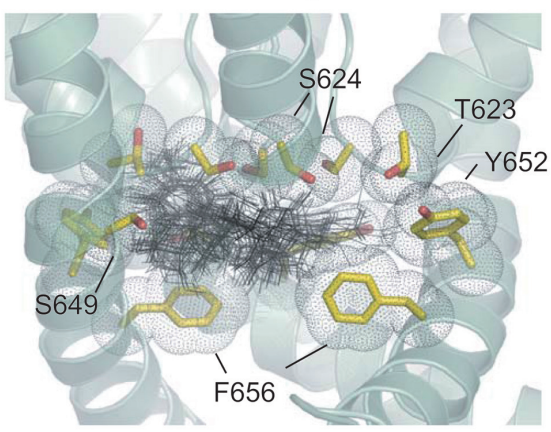

Membrane potential $(\mathrm{mV})$

b

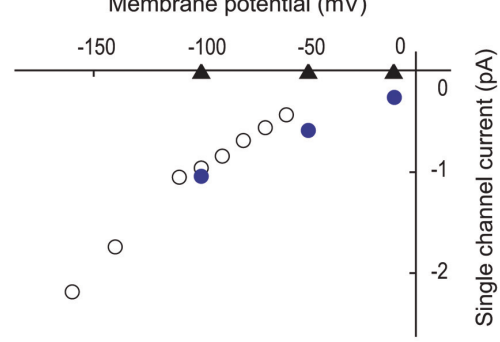

single channel current

\begin{tabular}{cccc} 
Voltage $(\mathrm{mV})$ & experiment $(\mathrm{pA})$ & $\mathrm{BD}$ simutation $(\mathrm{pA})$ \\
\hline & \multicolumn{4}{c}{ apo } & nifekalant-bound \\
-10 & -0.12 & -0.27 & 0.0 \\
-50 & -0.40 & -0.60 & 0.0 \\
-100 & -0.96 & -1.05 & 0.0
\end{tabular}

Fig. 3. Protein-based prediction of hERG current density. A. Open conformation of ion conduction unit of hERG. The homology model of open conformation of the hERG pore region was generated based on KvAP structure as a template (a). Nifekalant, class III antiarrhythmic agent, was docked onto this model. Forty different solutions developed by each simulation are superimposed in the hERG model (b) [92]. B. Analysis of inner diameters of $h E R G$. The inner surfaces of hERG models in the absence and the presence of nifekalant (left and right, respectively) were traced with blue. The constraint by nifekalant in right panel was colored by green. The inner diameters of apo-state (black), hERG models with 5 different nifekalant docked poses (gray), and averaged pore diameter of 100 different poses (red) were plotted against the ion conduction pathway (b). The position of the selectivity filter is indicated

with a bar. C. Brownian dynamics simulation. The membrane-embedded hERG model was subjected to the Brownian dynamics simulation (a). The $30 \mu \mathrm{s}$ simulation showed the ions passing through the pore (closed circles), which was comparable to the current amplitude measured experimentally (open circles) (retraced from Mitcheson et al. 2000 [100]). In the presence of nifekalant, there was no ion permeation (closed triangles) (b). pathway separating the central cavity from the extracellular solution. The central cavity consists of a $10 \AA$ spherical water-filled cavity about halfway through the membrane. Four S6 helices situated at the internal face of the channel make up another physical constraint for ion conduction. Bending these helices at their hinge points dilates the cross-sectional area surrounded by S6 helices and results in channel opening.

Since the ion conduction pathways of most $\mathrm{K}^{+}$channels share considerable amino acid sequence similarity, they can be considered to possess analogous molecular architecture. So although hERG possesses large $\mathrm{N}$ and $\mathrm{C}$ termini $[2,89]$, the crystal structure of a $\mathrm{K}^{+}$channel with an open pore is a suitable reference structure to generate the 3D model of an ion conduction unit of hERG [47, 48, 50, 90-92].
An open channel conformation of a hERG pore region was generated with the homology modeling program MODELLER 6 [55], using the crystal structure of KvAP as a template [84]. The membrane-spanning ion conduction unit of hERG (between positions 548 and 673) has $24 \%$ amino acid identity and $36 \%$ homology to the corresponding region of KvAP. The sequence alignment of KcsA, KvAP, and hERG was generated by T-Coffee [93] and then manually adjusted to increase the accuracy of homology modeling. Although an extracellular loop between S5 and pore helix (between positions 572 and 610) is proposed to be structured and involved in ion conduction in hERG [94], this loop was omitted for simplicity. After the refinement of the structure by molecular mechanics, the model had no dihedral angle violation in the Ramachandran plot, with the exception of distal termini 
of each subunit (Fig. 3Aa). In the homology model, all amino acids responsible for drug binding face the central cavity. Masetti et al. (2007) performed an MD simulation for the open conformation model of hERG based on the KvAP structure to examine the stability and the quality of its conformation [48]. They showed that the root mean squared fluctuation of the $\mathrm{C} \alpha$ positions was small during the simulation, indicating that the open conformation model is stable and potentially reflects one aspect of the open state of hERG.

4.2. Docking of ligands to the hERG homology model. To predict compound positions on hERG, several studies have docked hERG blockers in the central cavity of the pore [47-52]. We used a protein-ligand flexible docking program, Sievgene [62]. A class III antiarrhythmic drug, nifekalant, was employed to dock onto the hERG model $[92,95]$. The atomic charges of the ligand were determined by the restricted electrostatic point charge procedure using $\mathrm{HF} / 6-31 \mathrm{G}^{*}$-level quantum chemical calculations [96]. GAMESS (General atomic and molecular electronic structure system) [97] and Gaussian98 (revision A.9; Gaussian, Inc., Wallingford, USA) were used to perform the quantum chemical calculations. The atomic charges for the proteins were the same as those in AMBER (assisted model building with energy refinement) parm99 [96]. Since Thr623, Ser624, Tyr652, and Phe 656 are known to participate in the nifekalantinduced block of hERG [92, 98], we selected the docking space for nifekalant close to these residues. The program suggested 100 different poses of nifekalant as solutions of docking. In Fig. 3Ab, the geometrical arrangement of the compound is shown for 40 successive examples with the highest score. Nifekalant is situated in a pocket surrounded by Thr623, Ser624, Tyr652, and Phe656 and positions orthogonally to the ion conduction pathway. This characteristic position was also observed with another hERG blocker, dofetilide (data not shown), and proposed for other compounds by structure-based analyses [47, 50 52]. Multiple-docking poses of nifekalant maintained the association with aromatic residues of Tyr652 and Phe656, but the numbers of other residues, which interacted with it, varied between poses. Farid et al. (2006) reported similar compound-hERG associations in docking simulations with terfenadine, cisapride, MK-499, s-ibtilide, clofilium, and sertindole and its analogs [47]. On the other hand, if no constraints are given for the docking space for nifekalant during computation, compounds tended to be distributed along the ion conduction pathway as reported by Rajamani et al. (2005) [49] and Masetti et al. (2007) [48].

A closed conformation state model of hERG has been also used as a template for docking studies [45, 99]. By rotating the S6 helix of the closed conformation model, Perry et al. (2006) [45] suggested that most compounds position along the ion conduction pathway and that the geometrical arrangement of features are in good agreement with pharmacophore distribution predicted by a ligand-based approach [17]. MK-499 was reported to be retained in the central cavity, and hERG was in the closed state [100]. Therefore, whereas there is no experimental evidence about the structural difference between compound-free and compound-bound hERGs in the closed state, the docking poses of compounds in the closed state of hERG might represent some aspects of drug-hERG association.

The score value from the docking programs should be related to the binding affinity of a compound [7]. Du et al. (2007) [52] performed a docking study with an open conformation model of hERG and its blockers with the GOLD program [61], and then they assessed the link between the GoldScore for the best pose with the experimental $I C_{50}$ values by partial least square methods. The $r^{2}$ value was 0.60 , indicating a moderate relationship between GoldScore and experimental $I C_{50}$. Österberg and Åqvist (2005) [50], Rajamani et al. (2005) [49], and Farid et al. (2006) [47] performed MD simulations for compounds and the associated side chains to obtain the stable binding configuration between compounds and hERG. There was good agreement between experimental $I C_{50}$ values and either the binding free energies $[49,50]$ or the refined score values from the docking program [47]. The effect of the ion occupation state in the selectivity filter on the binding free energies was originally recognized by docking simulations of the local anesthetic bupivacaine and a model of Kv1.5 [101]. This effect is considered to be due to the electrostatic repulsion of ions in the filter and the positively charged nitrogen center of the docked compound. Österberg and Åqvist (2005) [50] compared the binding energies of compounds docked with hERG when the selectivity filter was occupied by $\mathrm{K}^{+}$ions in either 1010 or 0101 states; the binding free energy was lowest with the $1010 \mathrm{~K}^{+}$ion occupation state.

In the Sievgene program, the score was calculated by a virtual potential function with a soft repulsion potential to reduce structural noise [62]. A comparison of the scores for 100 binding poses indicated that the difference between them was not significant. Therefore it is difficult to determine which binding configurations reflect the actual interaction state by the inspection of score values. We applied the molecular mechanics method to minimize the energy potential of each of the 100 different binding configurations. This step revealed no clear relationship between the score values from the docking program and the minimized energy potentials of each binding configuration (data not shown). Since it is not realistic to trace an ion trajectory with BD simulations of every one of these 100 compound-hERG complexes, it was necessary to select a minimal number of binding configurations of hERG and nifekalant before moving on to analysis with BD simulation. 
Although the mechanism of how the association of compounds with hERG blocks ion flow through the channel is still ambiguous, one idea is that compounds located in the central cavity act as a physical constraint for ion permeation [6]. This suggests that analysis of pore dimensions of hERG docked with compounds could reveal criteria for which binding configurations should be analyzed by the BD simulation. The HOLE is a program that visualizes an internal surface of the pore and calculates the pore dimensions at the same time (Fig. 3Ba) [102]. Nifekalant is positioned in the central cavity differently in each docking pose, so the diameters of the cavity were diverse between the poses (Fig. 3Bb). Since the score value is believed to reflect the affinity between ligand and receptor protein, this value was used as a weighing factor for averaging the pore dimension of each binding configuration to calculate normalized diameters of the hERG pore in complex with nifekalant. The averaged root means square deviation (RMSD) of the pore dimension of each binding configuration with the normalized dimension was $0.229 \AA$. One docked model that was ranked 5 th out of 100 models on score value showed the lowest RMSD value $(0.157 \AA)$, and this model was subjected to BD simulation. The structures of nifekalant-hERG complex and nifekalant-free hERG were refined to minimize the energy with the cosgene module in the myPresto program [103].

4.3. BD simulation for ion conduction through the ligand-hERG complex. The BD method can simulate for a period long enough to determine statistically consistent estimations of the current flow through ion channels and to study the effects of a compound on the conduction properties of the channel. Prior to simulating the effect of nifekalant on the ion conduction of hERG, we set the environment of the system. The system contained multiple $\mathrm{K}^{+}$and $\mathrm{Cl}^{-}$ions $(150 \mathrm{mM})$ on either side of the membrane in volumes of $8.1 \times 10^{6} \mathrm{~nm}^{3}$. Protein was handled as a 3D volumetric mesh with a bin size for the grid of $0.5 \AA$. Electrostatic force in a continuum model composed of an electrostatic potential, a reaction field potential, and a potential between ions was precalculated by solving Poisson equations. Lennard-Jones AMBER parm96 [104] was used to calculate van der Waals force of the proteinion and ion-ion interactions. An external electric field to drive ions to move through the channel was applied in parallel to the z-axis. By comparing the measured ion flow through a single channel and the number of ions passing through the model, the Lennard-Jones parameter for $\mathrm{K}^{+}$in the selectivity filter was modified to $3.458 \AA$ (Fig. $3 \mathrm{Cb})$ so as to fit the experimental data. We assumed that $\mathrm{Cl}^{-}$does not permeate the selectivity filter due to hydration, and the Lennard-Jones parameter for $\mathrm{Cl}^{-}$in the selectivity filter was given by the sum of $\mathrm{Cl}^{-}$and two wateroxygen atoms, $5.484 \AA(1.948 \AA+1.768 \times 2 \AA)$.

The BD program was executed on an Itanium 2 Work- station. The CPU time to complete a simulation period of $30 \mu \mathrm{s}$ was $2 \times 30$ days. The nifekalant-hERG complex would not allow ions to pass through the channel.

4.4. Issues and resolutions. The main issue in this series of in silico experiments is the validity of the homology model. The comparison of amino acids of the inner helix of different $\mathrm{K}^{+}$channels reveals diversity in residues that are thought to be critical for function. For example, some voltage-dependent $\mathrm{K}^{+}$channels possess a Pro-XPro motif ( $\mathrm{X}$ represents any hydrophobic residue) in the middle of the inner helix, and other channels such as hERG and KvAP do not. Since Pro has an amino nitrogen cyclized with the side chain terminal carbon, this unique characteristic of Pro confers the structural distortion in helix formation known as the "proline kink," which is thought to be directly linked to the biological function of the protein [105]. The inner helix movement functions as a gate of $\mathrm{K}^{+}$channels [106]. Therefore, whereas the highly conserved structural architecture of the ion conduction unit of $\mathrm{K}^{+}$channels provides the essential linkage between mechanics and function, their detailed motions are probably different during voltage-dependent gating.

Since the $\mathrm{K}^{+}$channel consists of four subunits, the distribution of residues responsible for a compound-associated block exhibits a fourfold symmetry. Besides this highly symmetrical arrangement of residues, the water-filled cavity provides a widened binding site for compounds. This large volume of the binding pocket of hERG sharply contrasts to the ligand-binding site in receptor proteins, which is a crevice surrounded by structural elements. These two characters may underlie the ability of hERG to accommodate a large number of compounds and also to hinder the identification of a single binding configuration between compounds and the channel with docking analysis. It may also explain why a single ligand-based pharmacophore model cannot fully describe the interaction between compounds and hERG.

The association of a compound with a receptor protein can cause conformational changes not only in the side chains and the backbone of the protein, but also in the compound. This molecular mechanism is known as an induced fit, and it plays a critical role in the recognition process [107]. During docking analysis, binding spaces were searched for on fixed-side chain positions of hERG by generating conformations of compound $a b l i b$, and then slightly shifting its side-chain position by optimizing the interaction. Thus the application of an induced fit for the recognition system was limited in this step. This issue can be partially overcome by carrying out molecular mechanics calculations to minimize energy potential prior to the BD simulation. However, the BD simulation also treated hERG as a rigid receptor model. It will need the fortification of computational power and/or a novel theoretical development to accelerate the MD simulation process to address these issues and to describe the 
compound-hERG association and ion-hERG association in detail.

As mentioned above, several amino acids have been identified as participating in the compound-induced block of hERG. However, how the binding configuration of these amino residues and the compound actually causes a block of ion flux is not yet clear. The structurebased computational analyses of binding state suggest that the compounds associate through multiple contacts with aromatic residues $[47,91]$, and their nitrogen centers (classical hallmark of hERG blockers) position in the central cavity where a $\mathrm{K}^{+}$ion locates in the 1010 occupation state [50]. These observations suggest that the compoundhERG association might be separated into two different modes of interaction, either while it is docked to interact with hERG or while it blocks ion flow. Furthermore, a subset of hERG blockers, such as nifekalant [92], azimilide [108], and almokalant [109], not only block, but also increase hERG current at low membrane potentials by shifting its activation curve toward a direction of hyperpolarization. The latter phenomenon is called "facilitation," and it is thought to be based on the compound stabilizing the open channel configuration of hERG [92]. This also implies that even though there may be only a single hERG blocker molecule, there may be at least two different binding configurations: one preventing ion flux and another allowing ion flow. Since depolarizing prepulse is a prerequisite for the facilitation [92], voltage-dependent conformational changes in a central cavity of hERG and/or compounds may lead to this pharmacological effect. Besides the flow of $\mathrm{K}^{+}$ions, such voltage-dependent conformational change in the central cavity during the activation and the C-type inactivation is thought to have an effect on the voltage-dependent current block by compounds. Furthermore, the activation gating effectively modulates the kinetics of compound association. At the present time the structure-based analytic approach described above could not reproduce these experimental observations. Therefore multiple experimental 3D structures with different association states with compounds are required to overcome this issue. The establishment of a prediction system for the hERG current will be important for the realistic understanding of these diverse pharmacological actions of compounds.

\section{Conclusion}

Here we propose a procedure to predict the activity of compounds to block hERG current. Since this system is an ensemble of computational steps, the strategy to integrate these steps and the criterion evaluation to connect each step to the subsequent step are crucial for the construction of the system. An attempt to incorporate a protein-based approach in this prediction system has an obvious advantage to provide the opportunity to uncover the mechanisms underlying the pharmacological action of compounds on hERG, which cannot be addressed by experiments. Furthermore, since the structure-based approach enables the visualization of the interaction between compounds and hERG, it will aid the design of new compounds with less affinity for hERG or that allow ion flows even though they may be situated in the central cavity. Therefore the in silico prediction system is worthwhile not only for the evaluation of compounds to identify their risk, assess their dose, and characterize the mechanism of block of hERG, but also for effective drug design during the initial stages of their development. An executable platform would be required to run this system. However, prior to the design of such a platform, experimental data listing hERG blocker-like compounds and ion channel residues participating in the association between compounds and hERG should be accumulated and integrated with the knowledge obtained by computational studies to validate and improve this procedure.

We are grateful to Dr. lan Findlay (CNRS UMR 6542 Faculté des Sciences, Université de Tours, France) for his critical reading of this manuscript. We also thank Ms. Yuko Nakaie, Ms. Rika Kirai and Ms. Mami Yokokawa for secretarial assistance. This work was supported by the Leading Project for Biosimulation, "Development of models for disease and drug action," and the Global COE (Centers of Excellence) Program, "A center of excellence for an in silico medicine," from the Ministry of Education, Culture, Sports, Science and Technology in Japan to Yoshihisa Kurachi.

\section{REFERENCES}

1. Sanguinetti MC, Jiang C, Curran ME, Keating MT. A mechanistic link between an inherited and an acquired cardiac arrhythmia: HERG encodes the $\mathrm{I}_{\mathrm{kr}}$ potassium channel. Cell. 1995;81:299-307.

2. Keating MT, Sanguinetti MC. Molecular and cellular mechanisms of cardiac arrhythmias. Cell. 2001;104:569-80.

3. Sanguinetti MC, Tristani-Firouzi M. hERG potassium channels and cardiac arrhythmia. Nature. 2006;440:463-9.

4. Roden DM. Acquired long QT syndromes and the risk of proarrhythmia J Cardiovasc Electrophysiol. 2000;11:938-40.

5. Brown AM. Drugs, hERG and sudden death. Cell Calcium. 2004;35:543-7.

6. Sanguinetti MC, Mitcheson JS. Predicting drug-hERG channel interactions that cause acquired long QT syndrome. Trends Pharmacol Sci. 2005;26:119-24.

7. Kroemer RT. Structure-based drug design: docking and scoring. Curr Protein Pept Sci. 2007;8:312-28.

8. Reddy AS, Pati SP, Kumar PP, Pradeep HN, Sastry GN. Virtual screening in drug discovery - a computational perspective. Curr Protein Pept Sci. 2007:8:329-51.

9. Aronov AM. Predictive in silico modeling for hERG channel blockers Drug Discov Today. 2005;10:149-55.

10. Jalaie M, Holsworth DD. QT interval prolongation: and the beat goes on. Mini Rev Med Chem. 2005;5:1083-91.

11. Bains W, Basman A, White C. HERG binding specificity and binding site structure: evidence from a fragment-based evolutionary computing SAR study. Prog Biophys Mol Biol. 2004;86:205-33.

12. Stansfeld PJ, Gedeck P, Gosling M, Cox B, Mitcheson JS, Sutcliffe MJ. Drug block of the hERG potassium channel: insight from modeling. Proteins. 2007;68:568-80.

13. Recanatini M, Cavalli A, Masetti M. Modeling hERG and its interactions with drugs: recent advances in light of current potassium channel simulations. ChemMedChem. 2008;3:523-35.

14. Keserü GM. Prediction of $h E R G$ potassium channel affinity by traditional and hologram qSAR methods. Bioorg Med Chem Lett. 2003;13:2773-5.

15. Yoshida K, Niwa T. Quantitative structure-activity relationship studies on inhibition of HERG potassium channels. J Chem Inf Model. 2006;46:1371-8.

16. Song M, Clark M. Development and evaluation of an in silico model for hERG 
binding J Chem Inf Model. 2006:46:392-400.

17. Cavalli A, Poluzzi E, De Ponti F, Recanatini M. Toward a pharmacophore for drugs inducing the long QT syndrome: insights from a CoMFA study of HERG $\mathrm{K}^{+}$channel blockers. J Med Chem. 2002;45:3844-53.

18. Aronov AM, Goldman BB. A model for identifying HERG $K^{+}$channel blockers Bioorg Med Chem. 2004;12:2307-15.

19. Aronov AM. Common pharmacophores for uncharged human ether-a-go-gorelated gene (hERG) blockers. J Med Chem. 2006;49:6917-21.

20. Ekins S, Crumb WJ, Sarazan RD, Wikel JH, Wrighton SA. Three-dimensiona quantitative structure-activity relationship for inhibition of human ether-a-gogo-related gene potassium channel. J Pharmacol Exp Ther. 2002;301:427-34.

21. Pearlstein RA, Vaz RJ, Kang J, Chen XL, Preobrazhenskaya M, Shchekotikhin AE, Korolev AM, Lysenkova LN, Miroshnikova OV, Hendrix J, Rampe D. Characterization of HERG potassium channel inhibition using CoMSiA 3D QSAR and homology modeling approaches. Bioorg Med Chem Lett. 2003;13:1829-35.

22. Cianchetta G, Li Y, Kang J, Rampe D, Fravolini A, Cruciani G, Vaz RJ. Predictive models for hERG potassium channel blockers. Bioorg Med Chem Lett. 2005;15:3637-42

23. Dubus $E$, lijaali I, Petitet $F$, Michel A. In silico classification of $h E R G$ channel blockers: a knowledge-based strategy. ChemMedChem. 2006;1:622-30.

24. Seierstad M, Agrafiotis DK. A QSAR model of hERG binding using a large diverse, and internally consistent training set. Chem Biol Drug Des. 2006;67:284-96

25. Tobita M, Nishikawa T, Nagashima R. A discriminant model constructed by the support vector machine method for HERG potassium channel inhibitors. Bioorg Med Chem Lett. 2005;15:2886-90

26. Coi A, Massarelli I, Murgia L, Saraceno M, Calderone V, Bianucci AM. Prediction of $h E R G$ potassium channel affinity by the CODESSA approach. Bioorg Med Chem. 2006;14:3153-9.

27. Schneider G, Neidhart W, Giller T, Schmid G. "Scaffold-Hopping" by Topological Pharmacophore Search: A Contribution to Virtual Screening Angew Chem Int Ed Engl. 1999;38:2894-6.

28. Sheridan RP, Nilakantan R, Dixon JS, Venkataraghavan R. The ensemble approach to distance geometry: application to the nicotinic pharmacophore. J Med Chem. 1986;29:899-906

29. Bradley EK, Beroza P, Penzotti JE, Grootenhuis PD, Spellmeyer DC, Miller JL. A rapid computational method for lead evolution: description and application to alpha(1)-adrenergic antagonists. J Med Chem. 2000;43:2770-4.

30. Mason JS, Good AC, Martin EJ. 3-D pharmacophores in drug discovery. Curr Pharm Des. 2001;7:567-97.

31. Tsujishita $\mathrm{H}$, Hirono S. CAMDAS: an automated conformational analysis system using molecular dynamics. Conformational Analyzer with Molecula Dynamics And Sampling. J Comput Aided Mol Des. 1997;11:305-15

32. Cramer RD 3rd, Patterson DE, Bunce JD. Recent advances in comparative molecular field analysis (CoMFA). Prog Clin Biol Res. 1989;291:161-5.

33. Klebe $G$, Mietzner T. A fast and efficient method to generate biologically relevant conformations. J Comput Aided Mol Des. 1994:8:583-606.

34. Shih TM, Smith RD, Toro L, Goldin AL. High-level expression and detection of ion channels in Xenopus oocytes. Methods Enzymol. 1998;293:529-56.

35. Witchel HJ, Milnes JT, Mitcheson JS, Hancox JC. Troubleshooting problems with in vitro screening of drugs for QT interval prolongation using HERG $\mathrm{K}^{+}$ channels expressed in mammalian cell lines and Xenopus oocytes. J Pharmacol Toxicol Methods. 2002:48:65-80.

36. Zhou Z, Vorperian VR, Gong Q, Zhang S, January CT. Block of HERG potassium channels by the antihistamine astemizole and its metabolites desmethylastemizole and norastemizole. J Cardiovasc Electrophysiol. 1999;10:836-43

37. Kang J, Wang L, Chen XL, Triggle DJ, Rampe D. Interactions of a series of fluoroquinolone antibacterial drugs with the human cardiac $\mathrm{K}^{+}$channe HERG. Mol Pharmacol. 2001;59:122-6

38. Morgan TK Jr, Sullivan ME. An overview of class III electrophysiological agents: a new generation of antiarrhythmic therapy. Prog Med Chem. 1992;29:65-108

39. Kongsamut S, Kang J, Chen XL, Roehr J, Rampe D. A comparison of the receptor binding and HERG channel affinities for a series of antipsychotic drugs. Eur J Pharmacol. 2002;450:37-41.

40. Davie C, Pierre-Valentin J, Pollard C, Standen N, Mitcheson J, Alexander P, Thong B. Comparative pharmacology of guinea pig cardiac myocyte and cloned hERG $\left(\mathrm{I}_{\mathrm{Kr}}\right)$ channel. J Cardiovasc Electrophysiol. 2004;15:1302-9.

41. Chouabe C, Drici MD, Romey G, Barhanin J, Lazdunski M. HERG and KvLQT1/lsK, the cardiac $\mathrm{K}^{+}$channels involved in long QT syndromes, are targets for calcium channel blockers. Mol Pharmacol. 1998;54:695-703.

42. Fernandez D, Ghanta A, Kauffman GW, Sanguinetti MC. Physicochemical features of the HERG channel drug binding site. J Biol Chem 2004;279:10120-7.

43. Lees-Miller JP, Duan Y, Teng GQ, Duff HJ. Molecular determinant of high-affinity dofetilide binding to HERG1 expressed in Xenopus oocytes: involvement of S6 sites. Mol Pharmacol. 2000;57:367-74.

44. Mitcheson JS, Chen J, Lin M, Culberson C, Sanguinetti MC. A structural basis for drug-induced long QT syndrome. Proc Natl Acad Sci U S A 2000;97:12329-33.

45. Perry M, Stansfeld PJ, Leaney J, Wood C, de Groot MJ, Leishman D, Sutcliffe MJ, Mitcheson JS. Drug binding interactions in the inner cavity of HERG channels: molecular insights from structure-activity relationships of clofilium and ibutilide analogs. Mol Pharmacol. 2006;69:509-19.

46. Kramer C, Beck B, Kriegl JM, Clark T. A composite model for hERG blockade. ChemMedChem. 2008;3:254-65.

47. Farid R, Day T, Friesner RA, Pearlstein RA. New insights about HERG blockade obtained from protein modeling, potential energy mapping, and docking studies. Bioorg Med Chem. 2006;14:3160-73.

48. Masetti M, Cavalli A, Recanatini M. Modeling the hERG potassium channel in a phospholipid bilayer: Molecular dynamics and drug docking studies. J Comput Chem. 2007; 29:795-808.

49. Rajamani R, Tounge BA, Li J, Reynolds CH. A two-state homology model of the hERG K+ channel: application to ligand binding. Bioorg Med Chem Lett. 2005;15:1737-41.

50. Österberg F, Åqvist J. Exploring blocker binding to a homology model of the open $\mathrm{hERG} \mathrm{K}^{+}$channel using docking and molecular dynamics methods. FEBS Lett. 2005;579:2939-44.

51. Choe H, Nah KH, Lee SN, Lee HS, Lee HS, Jo SH, Leem CH, Jang YJ. A novel hypothesis for the binding mode of HERG channel blockers. Biochem Biophys Res Commun. 2006;344:72-8.

52. Du L, Li M, You Q, Xia L. A novel structure-based virtual screening model for the $\mathrm{hERG}$ channel blockers. Biochem Biophys Res Commun 2007:355:889-94

53. Raman $P$, Cherezov V, Caffrey M. The membrane protein data bank. Cell Mol Life Sci. 2006;63:36-51.

54. Baker D, Sali A. Protein structure prediction and structural genomics. Science. 2001;294:93-6

55. Martí-Renom MA, Stuart AC, Fiser A, Sánchez R, Melo F, Sali A. Comparative protein structure modeling of genes and genomes. Annu Rev Biophys Biomol Struct. 2000;29:291-325.

56. Laskowski RA, MacArthur MW, Moss DS, Thornton JM. PROCHECK: a program to check the stereochemical quality of protein structures. J Appl Crystallogr. 1993;26:283-91

57. Meng EC, Gschwend DA, Blaney JM, Kuntz ID. Orientational sampling and rigid-body minimization in molecular docking. Proteins. 1993;17:266-78.

58. Goodsell DS, Morris GM, Olson AJ. Automated docking of flexible ligands: applications of AutoDock. J Mol Recognit. 1996;9:1-5

59. Rarey M, Kramer B, Lengauer T, Klebe G. A fast flexible docking method using an incremental construction algorithm. J Mol Biol. 1996;261:470-89.

60. Wu G, Robertson DH, Brooks CL 3rd, Vieth M. Detailed analysis of gridbased molecular docking: A case study of CDOCKER-A CHARMm-based MD docking algorithm. J Comput Chem. 2003;24:1549-62.

61. Verdonk ML, Cole JC, Hartshorn MJ, Murray CW, Taylor RD. Improved protein-ligand docking using GOLD. Proteins. 2003;52:609-23.

62. Fukunishi Y, Mikami Y, Nakamura H. Similarities among receptor pockets and among compounds: analysis and application to in silico ligand screening. J Mol Graph Model. 2005;24:34-45.

63. Kuyucak S, Andersen OS, Chung SH. Models of permeation in ion channels. Rep Prog Phys. 2001;64:1427-72.

64. Karplus M, McCammon JA. Molecular dynamics simulations of biomolecules. Nat Struct Biol. 2002;9:646-52.

65. Eisenberg RS. From structure to function in open ionic channels. J Membr Biol. 1999;171:1-24.

66. Moy G, Corry B, Kuyucak S, Chung SH. Tests of continuum theories as models of ion channels. I. Poisson-Boltzmann theory versus Brownian dynamics. Biophys J. 2000;78:2349-63.

67. Graf P, Nitzan A, Kurnikova MG, Coalson RD. A dynamic lattice Monte Carlo model of ion transport in inhomogeneous dielectric environments: method and implementation. J Phys Chem B. 2000;104:12324-38.

68. Bernèche $S$, Roux B. Energetics of ion conduction through the $\mathrm{K}+$ channel. Nature. 2001;414:73-7. 


\section{In Silico Prediction Systems for Chemical Block of hERG}

69. Luzhkov VB, Åqvist J. $\mathrm{K}^{+} / \mathrm{Na}^{+}$selectivity of the KcsA potassium channel from microscopic free energy perturbation calculations. Biochim Biophys Acta. 2001;1548:194-202.

70. Bernèche $\mathrm{S}$, Roux $\mathrm{B}$. A microscopic view of ion conduction through the $\mathrm{K}^{+}$ channel. Proc Natl Acad Sci U S A. 2003;100:8644-8.

71. Bernèche $S$, Roux $B$. A gate in the selectivity filter of potassium channels. Structure. 2005;13:591-600.

72. Cooper K, Jakobsson E, Wolynes P. The theory of ion transport through membrane channels. Prog Biophys Mol Biol. 1985;46:51-96

73. Hille B. Ion channels of excitable membranes, Third Edition. 2001.

74. Hoyles M, Kuyucak S, Chung SH. Computer simulation of ion conductance in membrane channels. Phys Rev E. 1998;58:3654-61.

75. Chung SH, Allen TW, Hoyles M, Kuyucak S. Permeation of ions across the potassium channel: brownian dynamics studies. Biophys J. 1999;77:2517-33.

76. Chung SH, Allen TW, Kuyucak S. Conducting-state properties of the KcsA potassium channel from molecular and Brownian dynamics simulations. Biophys J. 2002;82:628-45.

77. Im W, Seefeld S, Roux B. A Grand Canonical Monte Carlo-Brownian dynamics algorithm for simulating ion channels. Biophys J. 2000;79:788-801.

78. Corry B, Hoyles M, Allen TW, Walker M, Kuyucak S, Chung SH. Reservoir boundaries in Brownian dynamics simulations of ion channels. Biophys $\mathrm{J}$. 2002;82:1975-84

79. Allen TW, Kuyucak S, Chung SH. Molecular dynamics estimates of ion diffusion in model hydrophobic and KcsA potassium channels. Biophys Chem. 2000;86:1-14.

80. Doyle DA, Lee A, Lewis J, Kim E, Sheng M, MacKinnon R. Crystal structures of a complexed and peptide-free membrane protein-binding domain: molecular basis of peptide recognition by PDZ. Cell. 1996;85:1067-76.

81. Morais-Cabral JH, Zhou Y, MacKinnon R. Energetic optimization of ion conduction rate by the $\mathrm{K}^{+}$selectivity filter. Nature. 2001;414:37-42

82. Zhou Y, Morais-Cabral JH, Kaufman A, MacKinnon R. Chemistry of ion coordination and hydration revealed by a $\mathrm{K}^{+}$channel-Fab complex at $2.0 \mathrm{~A}$ resolution. Nature. 2001;414:43-8.

83. Jiang Y, Lee A, Chen J, Cadene M, Chait BT, MacKinnon R. Crystal structure and mechanism of a calcium-gated potassium channel. Nature. 2002;417:515-22.

84. Jiang Y, Lee A, Chen J, Ruta V, Cadene M, Chait BT, MacKinnon R. X-ray structure of a voltage-dependent $K^{+}$channel. Nature. 2003;423:33-41.

85. Long SB, Campbell EB, Mackinnon R. Crystal structure of a mammalian voltage-dependent Shaker family K ${ }^{+}$channel. Science. 2005;309:897-903.

86. Long SB, Tao X, Campbell EB, MacKinnon R. Atomic structure of a voltagedependent $\mathrm{K}^{+}$channel in a lipid membrane-like environment. Nature. 2007:450:376-82

87. Kuo A, Gulbis JM, Antcliff JF, Rahman T, Lowe ED, Zimmer J, Cuthbertson J, Ashcroft FM, Ezaki T, Doyle DA. Crystal structure of the potassium channel KirBac1.1 in the closed state. Science. 2003;300:1922-6.

88. Nishida M, Cadene M, Chait BT, MacKinnon R. Crystal structure of a Kir3.1prokaryotic Kir channel chimera. EMBO J. 2007;26:4005-15.

89. Morais Cabral JH, Lee A, Cohen SL, Chait BT, Li M, Mackinnon R. Crystal structure and functional analysis of the HERG potassium channel $\mathrm{N}$ terminus: a eukaryotic PAS domain. Cell. 1998;95:649-55.

90. Pearlstein R, Vaz R, Rampe D. Understanding the structure-activity relationship of the human ether-a-go-go-related gene cardiac $\mathrm{K}^{+}$channel. A model for bad behavior. J Med Chem. 2003;46:2017-22

91. Sánchez-Chapula JA, Navarro-Polanco RA, Culberson C, Chen J, Sanguinetti MC. Molecular determinants of voltage-dependent human ether-a-go-go related gene (HERG) $\mathrm{K}^{+}$channel block. J Biol Chem. 2002;277:23587-95

92. Hosaka Y, Iwata M, Kamiya N, Yamada M, Kinoshita K, Fukunishi Y, Tsujimae K, Hibino H, Aizawa Y, Inanobe A, Nakamura H, Kurachi $Y$. Mutational analysis of block and facilitation of HERG current by a class III anti-arrhythmic agent, nifekalant. Channels. 2007;1:198-208

93. Notredame C, Higgins DG, Heringa J. T-Coffee: A novel method for fast and accurate multiple sequence alignment. J Mol Biol. 2000;302:205-17.

94. Tseng GN, Sonawane KD, Korolkova YV, Zhang M, Liu J, Grishin EV, Guy HR. Probing the outer mouth structure of the HERG channel with peptide toxin footprinting and molecular modeling. Biophys J. 2007;92:3524-40.

95. Nakaya H, Tohse N, Takeda Y, Kanno M. Effects of MS-551, a new class III antiarrhythmic drug, on action potential and membrane currents in rabbit ventricular myocytes. Br J Pharmacol. 1993;109:157-63.

96. Wang J, Cieplak P, Kollman PA. How well does a restrained electrostatic potential (RESP) model perform in calculating conformational energies of organic and biological molecules? J Comput Chem. 2000;21:1049-74.

97. Schmidt MW, Baldridge KK, Boatz JA, Elbert ST, Gordon MS, Jensen JH Koseki S, Matsunaga N, Nguyen KA, Su S, Windus TL, Dupuis M, Montgomery JA Jr. General atomic and molecular electronic structure system. J Comput Chem. 1993;14:1347-63.

98. Kamiya K, Niwa R, Mitcheson JS, Sanguinetti MC. Molecular determinants of HERG channel block. Mol Pharmacol. 2006;69:1709-16.

99. Moreno I, Caballero R, González T, Arias C, Valenzuela C, Iriepa I, Gálvez E, Tamargo J, Delpón E. Effects of irbesartan on cloned potassium channels involved in human cardiac repolarization. J Pharmacol Exp Ther. 2003;304:862-73

100. Mitcheson JS, Chen J, Sanguinetti MC. Trapping of a methanesulfonanilide by closure of the HERG potassium channel activation gate. J Gen Physiol. 2000;115:229-40.

101. Luzhkov VB, Nilsson J, Arhem P, Åvist J. Computational modelling of the open-state Kv 1.5 ion channel block by bupivacaine. Biochim Biophys Acta. 2003;1652:35-51.

102. Smart OS, Neduvelil JG, Wang X, Wallace BA, Sansom MS. HOLE: a program for the analysis of the pore dimensions of ion channel structural models. J Mol Graph. 1996;14:354-60, 76

103. Fukunishi $Y$, Mikami $Y$, Nakamura $H$. The filling potential method: a method for estimating the free energy surface for protein-ligand docking. J Phys Chem B. 2003:107:13201-10.

104. Kollman P, Dixon R, Cornell W, Fox T, Chipot C, Pohorille A. The development/application of a 'minimalist' organic/biochemical molecular mechanic force field using a combination of ab initio calculations and experimental data. In: van Gunsteren WF, Weiner PK, Wilkinson AJ, editors Computer simulation of biomolecular systems. Vol. 3. 1997. p. 83-96.

105. Vanhoof G, Goossens F, De Meester I, Hendriks D, Scharpé S. Proline motifs in peptides and their biological processing. FASEB J. 1995;9:736-44.

106. Jiang Y, Lee A, Chen J, Cadene M, Chait BT, MacKinnon R. The open pore conformation of potassium channels. Nature. 2002;417:523-6.

107. Koshland DE. Application of a Theory of Enzyme Specificity to Protein Synthesis. Proc Natl Acad Sci U S A. 1958:44:98-104

108. Carmeliet $E$. Use-dependent block and use-dependent unblock of the delayed rectifier $\mathrm{K}^{+}$current by almokalant in rabbit ventricular myocytes. Circ Res. 1993;73:857-68.

109. Jiang M, Dun W, Fan JS, Tseng GN. Use-dependent 'agonist' effect of azimilide on the HERG channel. J Pharmacol Exp Ther. 1999;291:1324-36

110. Ridley JM, Milnes JT, Witchel HJ, Hancox JC. High affinity HERG $K^{+}$channel blockade by the antiarrhythmic agent dronedarone: resistance to mutations of the $S 6$ residues Y652 and F656. Biochem Biophys Res Commun. 2004:325:883-91.

111. Tie H, Walker BD, Valenzuela SM, Breit SN, Campbell TJ. The heart of psychotropic drug therapy. Lancet. 2000;355:1825.

112. Walker BD, Singleton CB, Tie H, Bursill JA, Wyse KR, Valenzuela SM, Breit SN, Campbell TJ. Comparative effects of azimilide and ambasilide on the human ether-a-go-go-related gene (HERG) potassium channel. Cardiovasc Res. 2000;48:44-58.

113. Karle CA, Kreye VAW, Thomas D, RöckI K, Kathöfer S, Zhang W, Kiehn J. Antiarrhythmic drug carvedilol inhibits HERG potassium channels Cardiovasc Res. 2001;49:361-70.

114. Wang J, Della Penna K, Wang H, Karczewski J, Connolly TM, Koblan KS, Bennett PB, Salata JJ. Functional and pharmacological properties of canine ERG potassium channels. Am J Physiol Heart Circ Physiol. 2003;284:H256-67.

115. Ridley JM, Milnes JT, Hancox JC, Witchel HJ. Clemastine, a conventiona antihistamine, is a high potency inhibitor of the HERG $\mathrm{K}^{+}$channel. J Mol Cell Cardiol. 2006;40:107-18

116. Paul AA, Witchel HJ, Hancox JC. Inhibition of HERG potassium channel current by the class $1 \mathrm{a}$ antiarrhythmic agent disopyramide. Biochem Biophys Res Commun. 2001;280:1243-50.

117. Weerapura M, Hébert TE, Nattel S. Dofetilide block involves interactions with open and inactivated states of HERG channels. Pflugers Arch. 2002;443:520-31.

118. Zhou Z, Gong Q, Ye B, Fan Z, Makielski JC, Robertson GA, January CT. Properties of HERG channels stably expressed in HEK 293 cells studied at physiological temperature. Biophys J. 1998;74:230-41.

119. Witchel HJ, Pabbathi VK, Hofmann G, Paul AA, Hancox JC. Inhibitory actions of the selective serotonin re-uptake inhibitor citalopram on HERG and ventricular L-type calcium currents. FEBS Lett. 2002:512:59-66.

120. Milnes JT, Crociani O, Arcangeli A, Hancox JC, Witchel HJ. Blockade of 


\section{A. INANOBE et al.}

HERG potassium currents by fluvoxamine: incomplete attenuation by $S 6$ mutations at F656 or Y652. Br J Pharmacol. 2003;139:887-98

121. Teschemacher AG, Seward EP, Hancox JC, Witchel HJ. Inhibition of the current of heterologously expressed HERG potassium channels by imipramine and amitriptyline. Br J Pharmacol. 1999;128:479-85.

122. Ferrer-Villada T, Navarro-Polanco RA, Rodríguez-Menchaca AA, BenavidesHaro DE, Sánchez-Chapula JA. Inhibition of cardiac HERG potassium channels by antidepressant maprotiline. Eur J Pharmacol. 2006;531:1-8.

123. Paul AA, Witchel HJ, Hancox JC. Inhibition of the current of heterologously expressed HERG potassium channels by flecainide and comparison with quinidine, propafenone and lignocaine. Br J Pharmacol. 2002;136:717-29.

124. Thomas D, Wimmer AB, Wu K, Hammerling BC, Ficker EK, Kuryshev YA, Kiehn J, Katus HA, Schoels W, Karle CA. Inhibition of human ether-a-gogo-related gene potassium channels by alpha 1-adrenoceptor antagonists prazosin, doxazosin, and terazosin. Naunyn Schmiedebergs Arch Pharmacol. 2004;369:462-72

125. Ridley JM, Milnes JT, Benest AV, Masters JD, Witchel HJ, Hancox JC. Characterisation of recombinant HERG $\mathrm{K}^{+}$channel blockade by the Class la antiarrhythmic drug procainamide. Biochem Biophys Res Commun 2003;306:388-93

126. Numaguchi $H$, Mullins FM, Johnson JP Jr, Johns DC, Po SS, Yang IC, Tomaselli GF, Balser JR. Probing the interaction between inactivation gating and Dd-sotalol block of HERG. Circ Res. 2000;87:1012-8.

127. Zitron E, Kiesecker C, Scholz E, Lück S, Bloehs R, Kathöfer S, Thomas D, Kiehn J, Kreye VA, Katus HA, Schoels W, Karle CA. Inhibition of cardiac HERG potassium channels by the atypical antidepressant trazodone. Naunyn Schmiedebergs Arch Pharmacol. 2004;370:146-56.

128. Su Z, Chen J, Martin RL, McDermott JS, Cox BF, Gopalakrishnan M, Gintant GA. Block of hERG channel by ziprasidone: biophysical properties and molecular determinants. Biochem Pharmacol. 2006;71:278-86.

129. Scholz EP, Zitron E, Kiesecker C, Lueck S, Kathöfer S, Thomas D, Weretka S, Peth S, Kreye VA, Schoels W, Katus HA, Kiehn J, Karle CA. Drug binding to aromatic residues in the HERG channel pore cavity as possible explanation for acquired Long QT syndrome by antiparkinsonian drug budipine. Naunyn Schmiedebergs Arch Pharmacol. 2003;368:404-14. 